\title{
Estudio de las competencias digitales en el espectador fan español
}

Recibido: 2017-01-19

Enviado a pares: 2017-01-20
DOI: $10.5294 /$ pacla.2017.20.4.4

Aprobado por pares: 2017-04-12

Aceptado: 2017-05-05

\section{Para citar este artículo / to reference this article / para citar este artigo}

Lozano Delmar, J., Herrero-Diz, P., Del toro, A. \& Sánchez-Martín, M. 2017. Estudio de las competencias digitales en el espectador fan español. Palabra Clave 20(4), 917-947.

DOI: 10.5294/pacla.2017.20.4.4

\section{Resumen}

Hoy existen múltiples estudios que abordan el análisis de las culturas participativas y, más específicamente, el espectador fan de películas o series de televisión. Henry Jenkins, huyendo del estereotipo de brainless consumers y social misfits que dedican su vida a cultivar un "conocimiento inútil", marca un punto de inflexión al plantear un perfil de fan que se corresponde con personas cultas. Desde este momento, los fan studies están viviendo su época dorada con diferentes investigadores o aca-fans que estudian y analizan el fenómeno en sus diferentes países de origen. El objetivo general de esta investigación es realizar una radiografía, estudio y descripción del espectador fan español en relación con sus competencias digitales. Ade-

\footnotetext{
orcid.org/0000-0002-8708-1004. Universidad Loyola Andalucía, España. pherrero@uloyola.es orcid.org/0000-0002-0974-1118. Universidad Loyola Andalucía, España. jlozano@uloyola.es

3 orcid.org/0000-0002-0919-3238. Universidad Loyola Andalucía, España. atoro@uloyola.es

4 orcid.org/0000-0002-7387-9971. Universidad Loyola Andalucía, España. msanchez@uloyola.es
} 
más, se persiguen dos objetivos secundarios: realizar una comparativa entre espectadores fan y espectadores no fan de acuerdo con sus competencias digitales y analizar el grado de correlación existente entre el nivel de competencias digitales del espectador fan y el número de series y películas del que se considera fan. Para abordar estos objetivos, se administró un cuestionario en línea elaborado ad hoc, en el que participaron 1003 consumidores de ficción audiovisual, cuyos datos se analizaron utilizando una metodología cuantitativa. Los análisis descriptivos y comparativos nos permitieron concluir que, en primer lugar, la práctica que dominan los espectadores fans es la búsqueda de información. En segundo lugar, que el espectador fan tiene mayores competencias digitales que el espectador no fan. Y, por último, que el nivel de competencias en los ítems de interacción y edición de contenidos en los fans es directamente proporcional al número de productos de los que el espectador se considera fan.

\section{Palabras clave}

Fan; competencias digitales; prosumidor; audiencias activas (Fuente: Tesauro de la Unesco). 


\section{Study of Digital Skills in Spanish Fans}

\section{Abstract}

There are several studies nowadays that address the analysis of participatory cultures and, more specifically, the fan audience of movies or television series. Henry Jenkins, moving away from the stereotype of brainless consumers and social misfits who dedicate their lives to developing "useless knowledge," marks a turning point by suggesting a fan profile that corresponds to educated people. From this moment, fan studies are living their golden age with different researchers or aca-fans who study and analyze the phenomenon in their different countries of origin. The general objective of this research is to take an x-ray, study and describe the Spanish fan audience regarding their digital skills. There are also two secondary objectives: to make a comparison between fan audience and non-fan audience according to their digital skills and to analyze the correlation degree between the level of digital skills of the fan audience and the number of series and movies of which they consider themselves to be fans. To address these objectives, an ad hoc online questionnaire was administered, involving 1003 audiovisual fiction consumers, whose data were analyzed using a quantitative methodology. Descriptive and comparative analyzes allowed us to conclude that, first of all, the practice that fan audience control is the search for information. Second, the fan audience has greater digital skills than nonfan audience. And, finally, that the level of skills in the content interaction and editing elements in fans is directly proportional to the number of products of which the audience consider themselves to be fans.

\section{Keywords}

Fan; digital skills; prosumer; active audience (Source: Unesco Thesaurus). 


\section{Estudo das habilidades digitais no espectador fã espanhol}

\section{Resumo}

Hoje existem múltiplos estudos que abordam a análise das culturas participativas e, mais especificamente, o espectador fã de filmes ou séries de televisão. Henry Jenkins, afastando-se do estereótipo de brainless consumers e social misfits que dedicam a sua vida a cultivar um "conhecimento inútil", marca um ponto de inflexão ao abordar um perfil de fã que corresponde a pessoas cultas. Desde este momento, os fan studies estão vivendo a sua época dourada com diferentes pesquisadores ou aca-fans que estudam e analisam o fenômeno em seus diferentes países de origem. $\mathrm{O}$ objetivo geral desta pesquisa é realizar uma radiografia, estudo e descrição do espectador fã espanhol com relação às suas habilidades digitais. Além disso, se perseguem dois objetivos secundários: realizar uma comparativa entre espectadores fã e espectadores não fã de acordo às suas habilidades digitais e analisar o grau de correlação existente entre o nível de habilidades digitais do espectador fã e o número de séries e filmes do qual se considera fã. Para abordar estes objetivos, foi aplicado um questionário online elaborado ad hoc, no qual participaram 1003 consumidores de ficção audiovisual, cujos dados foram analisados utilizando uma metodologia quantitativa. As análises descritivas e comparativas nos permitiram concluir que, em primeiro lugar, a prática que os espectadores fãs dominam é a busca de informação. Em segundo lugar, que o espectador fã tem mais habilidades digitais do que o espectador não fã. E, por último, que o nível de habilidades nos itens de interação e edição de conteúdos nos fãs é diretamente proporcional ao número de produtos dos que o espectador se considera fã.

\section{Palavras-chave}

Fã; habilidades digitais; prossumidor; audiências ativas (Fonte: Tesauro da Unesco). 


\section{Introducción: la alfabetización mediática en las audiencias activas}

El debate sobre la alfabetización mediática de las audiencias es una constante por la actualización permanente de nuevas formas de participar en los medios de comunicación, la aparición de nuevas herramientas, formatos o narrativas y por la incorporación de usuarios cada vez más jóvenes, con distintos intereses y lenguajes. En este escenario, que Ferrés i Prats y Masanet (2015, p. 14) califican de "caos terminológico" (educación en medios, educación para los medios, educomunicación, educación en comunicación audiovisual, alfabetización mediática, media literacy, educación mediática, multialfabetización, alfabetización informacional, nuevas alfabetizaciones, alfabetización digital, alfabetizaciones múltiples, transliteracy), resulta difícil establecer una definición imperecedera sobre qué es la alfabetización mediática y qué competencias debe integrar para formar a una audiencia activa, crítica y responsable en sus intervenciones, aunque sabemos que el concepto competencia mediática implica los lenguajes, la tecnología, los procesos de producción y difusión, los procesos de recepción e interacción, la ideología y los valores y la dimensión estética (Ferrés i Prats, Aguaded-Gómez y García-Matilla, 2012, p. 25). Estos autores reivindican, por ello, una alfabetización mediática integradora ante la inherente "experiencia mediática" (Ferrés i Prats y Masanet, 2015, p. 18) de cualquier interlocutor que consume y crea mensajes y que rompe con conceptos tradicionales, como sociedad de la información y sociedad del conocimiento, y lo sitúa como audiencia dentro una nueva sociedad: la sociedad del entretenimiento.

Entre la variedad de propuestas que existen y buscando el mayor consenso internacional, los principales expertos (Bergsma et al., 2007) pertenecientes a la Asociación Nacional de Educación en Alfabetización Mediática en los Estados Unidos, conocida como NAMLE (National Association of Media Literacy Education), han desarrollado seis principios básicos, esenciales, bajo el título Core principles o media literacy education, en torno a tres factores: 1) el pensamiento crítico, 2) los indicadores culturales y 3 ) la voz de los individuos (Mihailidis, 2014, p. 35). 
De acuerdo con el punto cuatro de los core principles, "La alfabetización mediática desarrolla participantes informados, reflexivos y comprometidos, capacidades esenciales para una sociedad democrática", Mihailidis (2014) afirma que existe un ciudadano activo que participa de los medios y que, a mayor formación, es decir, cuanto más competente sea digitalmente (más alfabetizado en medios), mejor ciudadano será en su comportamiento en internet. Para ello, elabora un modelo de representación del nuevo contexto de convivencia entre los ciudadanos (usuarios) y los medios, en el que debe haber un acuerdo entre estos medios de masas y el público para garantizar una participación responsable y democrática. Pues una sociedad es más libre a través de la participación en sus sistemas de expresión y es la alfabetización mediática, la educación, tanto en espacios formales como informales de aprendizaje, la única vía para construirla. Esta visión coincide con la de Frau-Meigs (2014), quien aboga por el término transalfabetización —alfabetizaciones informacional, mediática e informática - como la composición perfecta de distintas competencias para formar a usuarios inteligentes, responsables y generosos en el uso de los medios.

En el centro del modelo de Mihailidis (2014), en el que comparten atmósfera los ciudadanos y los medios, el autor sitúa las herramientas de la participación (participatory tools) que permiten la creación de contenidos en múltiples formatos y lenguajes y la posibilidad de compartirlos. Con ellas, convergen, por un lado, las plataformas móviles (mobile platforms) o los teléfonos y dispositivos inteligentes, y en otra esfera, la distribución (spreadability), que trabaja para combatir la distancia entre los medios y los ciudadanos. Su trabajo define, por tanto, a un nuevo ciudadano emergente (the emerging citizen), comprometido, que participa en la cultura digital. En él, recoge todas las teorías de referencia existentes sobre alfabetización mediática (media literacy) y las competencias digitales en las multitudes (crowds) en general. Entre ellas, destaca el paradigma de Shirky (2010) por su dimensión social, el carácter participativo y el compromiso que encuentra en las audiencias activas.

Mihailidis (2014, pp. 61-64) identifica en su propuesta cinco competencias esenciales para un sujeto alfabetizado en medios que participa de la 
cultura en red, The C's of media literacy networks: 1) curate (curación) o la capacidad de organizar, compartir ideas, enlaces, contenidos en general, expresarse, agregar, mejorar, ampliar o corregir; 2) critique (capacidad crítica) o facilitar el pensamiento y el análisis crítico en la red; 3 ) Contribute (contribuir, participar o aportar) o compartir información y crear significados en diferentes formatos y en distintos canales de participación, de manera productiva, tan simple como comentar, postear o distribuir; 4) collaborate (colaborar) o cómo aportar contenidos de valor; y 5) create (crear) o la facultad para producir, compartir y apropiarse de los contenidos de los medios en los espacios públicos.

Finalmente, el profesor formula su propio modelo, llamado "Las 5 aes de la alfabetización mediática para los ciudadanos emergentes" (The 5 A's of media literacy for the emerging citizen): 1) access (acceso), 2) awareness (conciencia), 3) assessment (valoración), 4) appreciation (apreciación) y 5) action (acción), para dar respuesta a un usuario formado que presenta nuevas competencias (Mihailidis, 2014). Y concluye que, en general, los ciudadanos alfabetizados en los medios de comunicación consideran su participación como una aportación a la escena pública, donde ofrecen algo a una audiencia activa y promueven los temas de los que se habla (la agenda).

Partiendo de este contexto, esta investigación conecta los estudios sobre alfabetización mediática y competencias digitales con los de audiencias activas o fans, pues afirma Jenkins (2006), y lo veremos más adelante, es necesario conectar el aprendizaje con la experiencia activa. $\mathrm{Si}$, como dice Mihailidis (2014), la cultura participativa reconoce que las audiencias desempeñarán un papel importante en la participación de otros o en el rechazo de una idea, deberíamos ser capaces de encontrar estas competencias en las audiencias activas o fans (p. 156). Ello debe hacerse, además, desde las primeras etapas de la participación que revela una intervención cada vez más temprana. Aguaded y Urbano-Cayuela (2014, p. 137) hablan de un nuevo "prosumidor infantil mediático-televisivo" que participa de los medios creando contenidos desde pequeño, consciente de la capacidad de distribución de internet y reivindican, por tanto, la importancia de la puesta en marcha de un nuevo modelo de enseñanza-aprendizaje de la alfabetización mediática. 


\title{
Web 2.0, democratización del contenido y fandom digital
}

Los trabajos de Lisa A. Lewis y H. Jenkins en 1992 marcaron un punto de inflexión importante en lo que se conoce hoy en día como fan studies. Ambos estudios criticaban duramente los estereotipos y clichés construidos en torno a los fans y los redefinían como unos actores y creadores activos en lo que posteriormente Jenkins definiría como convergence culture o cultura de la convergencia (2006). Esta cultura de la participación es un cambio de la distribución a la circulación, un modelo de cultura "que considera al público no solo como consumidores de mensajes preconstruidos, sino como personas que están dando forma, compartiendo, re-enmarcando y remezclado el contenido de los medios de una manera que antes era quizás inimaginable" (Jenkins, Ford y Green, 2015, p. 26).

Como indicaba el propio Jenkins en Textual Poachers: Television fans and participatory culture (1992), el concepto estereotipado del fan, aunque tiene una base objetiva limitada, es una proyección de las preocupaciones sobre la violación de las jerarquías culturales dominantes. La cultura de los fans desdibuja estos límites al tratar los textos populares como si merecieran la misma atención y reconocimiento que los textos canónicos. Jenkins, a partir de investigaciones etnográficas de audiencias, pondría el acento sobre la intensidad de la implicación emocional e intelectual de determinados receptores mediáticos:

\begin{abstract}
Mirar la televisión como fan implica diferentes niveles de atención y pone en marcha unas capacidades diferentes de las de un espectador ocasional que ve el mismo programa [...] Además, esta recepción no se da de forma aislada, sino que se estructura a través de la contribución de otros fanes, motivada por un deseo de interaccionar con una comunidad cultural y social más amplia. (pp. 76 y 97)
\end{abstract}

Con esta obra, Jenkins sentaría las bases de la redefinición del fan (mediático) como un productor activo y manipulador de significados (1992, p. 37). Esta primera ola en los fan studies fue seguida de un fortalecimiento gracias a las contribuciones académicas, entre otros, de Hills (2002), Sandvoss (2005), Gray, Sandvoss y Harrington (2007) y Hellekson y Busse (2014). 
Al mismo tiempo, la revolución digital ha tenido un profundo impacto en el fandom, empoderando y desempoderando, borrando las líneas entre productores y consumidores, creando relaciones entre corporaciones y fans y abanderando nuevas formas de producciones culturales (Pearson, 2010, p. 84). Aunque, como el propio Jenkins (1992) indica, las comunidades fan y sus prácticas participativas ya existían previamente a este contexto digital, internet sí que ha convertido las acciones del fandom en unas prácticas de escala global. En esta línea, es interesante apuntar los comentarios de Paul Booth, en su entrevista con la investigadora María del Mar Grandío sobre fans y series de televisión: "Los fans se quieren reunir en formas más íntimas de las que permite la tecnología. La tecnología digital ha abierto realmente el mundo del fandom [... $]$ Sin embargo, para muchos fans, el cara a cara es la mejor manera de interactuar con los demás" (2016, p. 151).

Bennett (2014) concreta explicando los cambios fundamentales que la cultura y las herramientas digitales han producido en las prácticas de las comunidades fans. De este modo, para la autora, la praxis digital ha modificado, principalmente, la comunicación entre comunidades, la creatividad, el conocimiento compartido y la organización y empoderamiento ciudadano; al igual que lo consideran Jenkins (2006) y Mihailidis (2014), quienes observan este fenómeno como una oportunidad poderosa por lo que supone para las audiencias formar parte del ecosistema de los medios.

Para entender la evolución de internet y el papel que asumen los usuarios y, por ende, los fans, resulta necesario comprender previamente el concepto de web 2.0 acuñado por O'Reilly (2005) y sus posteriores implicaciones en el contexto de la convergence culture definida por Jenkins. Una idea, la de web participativa o web 2.0, que para diversos autores (Cobo y Pardo, 2007; Kaplan y Haenlein, 2010; Aced, 2010; Dans, 2010) supone una de las principales revoluciones en el ámbito de la comunicación, ya que implica que internet ya no es simplemente un escaparate de contenidos, sino que se ha convertido en una plataforma abierta basada en la participación y, por tanto, convergencia de los usuarios. 
En este sentido, Kaplan y Haenlein (2010) apuntan que la web 2.0 es la plataforma donde los contenidos ya no son creados por un grupo pequeño de individuos, sino que están modificados y elaborados por el conjunto de los usuarios de manera colaborativa. De la misma forma, Ritzer y Jurgenson (2010) afirman que en la web 2.0 el contenido es generado por el usuario, frente a la web tradicional o 1.0, donde es aportada por un proveedor. Por su parte, Dhar y Chang (2009) señalan que este nuevo internet ha terminado con la hegemonía de los creadores de contenidos más tradicionales como fuentes primarias de información.

No solo esto, esta web 2.0 supone un paso tecnológico previo necesario que permitió el posterior desarrollo de los medios sociales. Una tecnología que lleva a un siguiente nivel la participación de los usuarios. Así, Kietzmann, Hermkens, McCarthy y Silvestre (2011) destacan que los medios sociales digitales son plataformas que aprovechan la tecnología de la web para fomentar la creación, modificación y distribución del contenido generado por el usuario. Siguiendo esta idea, a partir de su análisis, Hays, Page y Buhalis (2013) concluyen que los medios sociales hacen referencia a comunidades en línea "participativas", "coloquiales" y "fluidas", centradas en el usuario y en el contenido que genera.

\section{Competencias digitales y fandom: búsqueda de información, interacción y edición de contenido}

En este entorno digital, el fan se ha convertido en prosumidor digital. Este concepto fue anticipado por McLuhan y Nevitt en 1972, cuando apuntan que las nuevas tecnologías permitirían al consumidor asumir los roles de productor y consumidor de contenidos al mismo tiempo (Islas y Arribas, 2010). No obstante, fue Alvin Toffler quien propuso expresamente el término en 1980 con su libro La tercera ola, defendiendo que estos prosumidores adquieren una importancia creciente que determinan el rumbo de la "economía invisible" (Ritzer y Jurgenson, 2010). Según Alvin y Heidi Toffler (1980), el término se refiere al hecho de que creamos bienes, servicios o experiencias para nuestro propio uso o disfrute, en lugar de para venderlos o intercambiarlos (Islas y Arribas, 2010). Estos prosumidores son los líderes de la cultura de la convergencia de Jenkins y para algunos 
autores, como Van Dijck (2009), estos no solo impactan en internet, sino que, además, lideran una transformación económica, social y cultural en el ámbito global.

Una de las prácticas con las que se asocia al prosumidor es el user generated content (UGC). De acuerdo con Kaplan y Haenlein (2010), el término UGC puede ser considerado como el conjunto de todas las formas en las que los usuarios hacen uso de los medios sociales. El UGC protagoniza buena parte de las interacciones que realizan los usuarios a través de la web 2.0 y los medios sociales; ejemplo de ello son los blogs y las redes sociales, que permiten a los usuarios publicar y acceder a la información de manera sencilla (Dhar y Chang, 2009). Según estos autores, como consecuencia de esto, la proporción de contenido que ha sido generado por los usuarios que encontramos en internet aumenta progresivamente y cubre poco a poco todas las temáticas posibles. Si bien es cierto que son las personas las que contribuyen de manera individual en mayor medida a la creación de este UGC, cada vez encontramos ejemplos más relevantes de grupos de personas que trabajan de forma colaborativa (Ransbotham, Kane y Lurie, 2012).

En términos generales, la participación de las audiencias activas y los fans se ha venido vinculando a este UGC. Como explican Zubernis y Larsen (2012, p. 16), los fans casi siempre han sido categorizados por los académicos en función de su nivel de participación y, en muchos casos, reducen esta participación a la producción concreta de contenido (independiente de su naturaleza). Los fan studies llevan ya un tiempo intentando definir la identidad del fan a través de su comportamiento. Fiske (1992) fue uno de los primeros en proponer un modelo que analizaba la productividad del fan de acuerdo con tres niveles: 1) producción semiótica, 2) enunciativa y 3$)$ textual. En esta última, se producía el contenido como tal creado por los fans. Tras esto, Abercrombie y Longhurst (1998) establecieron su propia taxonomía de participación de las audiencias e instituyeron un audience continuum, marcado por los siguientes roles: consumers, fans, cultists, enthusiastsy pretty producers. Para estos autores, la producción textual de Fiske (1992) adquiriría mayor importancia conforme se avance en el continuum, 
hasta llegar a los productores (pretty producers). En este último escalón, es donde se producía realmente la creación de contenido. Estos autores también dialogaron sobre competencias y habilidades en función del tipo de audiencia: técnicas, analíticas e interpretativas (1998).

A estas categorizaciones y taxonomías se unió la propuesta por el usuario Obsession_inc en 2009. Como indican Hellekson y Busse (2014), hoy en día los fan studies parecen haber adoptado esta catalogación y consideran principalmente dos tipos de fans:

Affirmative fans tend to collect, view, and play, to discuss, analyze, and criticize. Transformative fans, however, take a creative step to make the worlds and characters their own, be it by telling stories, cosplaying the characters, creating artworks, or engaging in any of the many other forms active fan participation can take. ${ }^{5}$ (pp. 3-4)

Así pues, el debate académico en torno a la identidad fan es un concepto líquido que va mutando y cambiando al mismo tiempo que va avanzando la sociedad digital y las prácticas sociales de la web 2.0. El propio Hills (2013), por un lado, reflexiona sobre la pertinencia de la catalogación de Fiske hoy en día, en un mundo posinternet y pone en cuestión qué debe o no considerarse producción textual: "Comentarios, tweets o cambios de estado pueden todos ser potencialmente formas de productividad textual fan". Por otro lado, Jenkins (2014) responde a la taxonomía de Obsession_inc diciendo que los affirmative fans pueden verse también como transformative fans, si se tiene en cuenta que muchas formas de especulación y crítica fan son también transformational: potencian nuevas formas de pensar y relacionarse con el universo ficcional. Así pues, la insistencia en considerar estrictamente la creación o edición de contenidos como la práctica fundamental de la cultura participativa es una afirmación arriesgada y limitada que desmerece otros tipos de participación, como la evaluación, la valoración, la crítica y la redistribución del material (Jenkins, Ford y Green, 2015, p. 178).

5 Los affirmative fans tienden a coleccionar, ver, jugar, discutir, analizar y criticar. Los transformative fans, por otro lado, dan un paso creativo más para hacer los mundos y personajes suyos propios, ya sea contando historias, cosplaying los personajes, creando diferentes tipos de artworks o implicándose en cualquier otra forma de participación fan. 
Como explican Zubernis y Larsen, "un número importante de fans definiría su participación en términos de consumo activo de información $[\ldots]$ Los fans construyen, participan, se encuentran los unos a los otros $[\ldots] \mathrm{e}$ interactúan directamente con actores, escritores y otros agentes de la industria" (2012, p. 16). En definitiva, los fans raramente se comprometen en una única práctica (p. 17) y la producción de contenido es igualmente importante para la definición de la identidad fan como la búsqueda de información y la interacción.

Jason Mittell (2013) habla de un fandom forense que disecciona las complejas narrativas seriales que se están creando hoy día para la pequeña pantalla. El panorama digital no solo está potenciando la aparición de nuevas narrativas que se expanden a través de diferentes formatos y medios. De este modo, las narrativas transmedia y otras narrativas envolventes promueven compromisos de espectadores activos a largo plazo, que diseccionan y saborean las tramas en foros en línea y fuera de línea y otros espacios de internet. Mittell (2013) complementa el término "propagable" (spreadable) de Jenkins, Ford y Green (2015) con el de "profundizable" (drillable): los textos profundizables pasan a ser propagables a partir de los procesos de recolección de información y búsqueda de significados, interpretando, contextualizando (p. 159). En esta línea, son interesantes las investigaciones de Guerrero (2015) sobre los efectos de las narrativas transmedia en la creación y el consumo de fan fictions y las competencias interpretativas de estos fans.

Igualmente, el propio consumo televisivo se ha visto transformado radicalmente. Respecto de series televisivas, Cascajosa (2016) habla de "cultura de las series". Para la autora, "las nuevas tecnologías no solo han dado a los fans de las series nuevas opciones para verlas, sino también un elemento básico para el desarrollo de otras actividades en torno a ellas, como socializar" (p. 183). Además, aparecen nuevos conceptos como la televisión social: una forma de televisión interactiva que se caracteriza por la participación e interacción de los espectadores con los contenidos, que actúan como audiencia social, gracias a los medios sociales y usando para ello 
dispositivos de segunda pantalla (Quintas y González , 2015). Como vemos, esta televisión social está estrechamente vinculada al fenómeno de la doble pantalla, es decir, el acceso a internet mediante teléfonos inteligentes, tabletas u otros dispositivos de forma simultánea al consumo de televisión. Esta doble pantalla se ha convertido en un objeto de análisis cada vez más extendido en los estudios sobre audiencias. De acuerdo con los datos de 2016, de la AIMC, $41.9 \%$ de los internautas españoles se conecta a internet a través de otros dispositivos mientras ve la televisión de manera frecuente y $31.8 \%$ lo hace de manera ocasional. De estos usuarios que simultanean televisión e internet, $20.2 \%$ ve comentarios sobre el programa de televisión, $10.7 \%$ realiza comentarios, $15 \%$ utiliza el hashtag del programa, $11.2 \%$ visita la web o la aplicación del programa y $35.9 \%$ busca información en internet sobre él. Para ello, según la AIMC, hace especialmente uso del teléfono inteligente $57.2 \%$, seguido del ordenador con $25.5 \%$ y de tabletas con $16.6 \%$. Por tanto, estos datos avalan el fenómeno creciente de la participación de los espectadores en internet de forma simultánea al consumo de televisión. En definitiva, la búsqueda y exploración de información relacionada con el producto audiovisual y la interacción — simultánea o no-con otros espectadores puede ser igualmente relevante que la producción o edición de contenido.

Por último, también es interesante destacar el reciente trabajo de Grandío (2016), quien, en su estudio sobre los fans de series televisivas, establece varios niveles de fans basados en sus acciones sociales una vez se produce la conexión con el producto ficcional:

1. Fans buscadores extratextuales: estos seguidores de la serie no se limitan a consumir los episodios sino que tienen una necesidad de conocer más sobre la serie, en busca de información relacionada principalmente a través de internet.

2. Fans participativos-conversadores: debido a nuevas tendencias como la televisión social, el impacto de las nuevas tecnologías y las redes sociales han conseguido que la experiencia televisiva sea más entretenida y colectiva que nunca. 
3. Fans participativos-creadores: aquí se ubicarían los fans prosumidores que generan creaciones artísticas como fan arts, fan vid, memes o fan fictions.

Como puede observarse, estas definiciones de Grandío coinciden en una visión del fan que no solo edita o genera contenido, sino que, además, busca información e interactúa con otros fans a través de medios sociales en un entorno digital. Además de estos niveles, Grandío (2016) profundiza en un cuarto nivel: los fans participativos-activistas, que se mueven en acciones comunes para solicitar, por ejemplo, renovaciones de series canceladas.

Teniendo todo esto en cuenta, el objetivo principal de este trabajo es realizar una radiografía, estudio y descripción del espectador fan español en relación con sus competencias digitales que parta de estas tres dimensiones discutidas: 1) búsqueda de información, 2) interacción y 3 ) edición de contenido. Además de este objetivo principal, el trabajo persigue dos objetivos secundarios. En primer lugar, realizar una comparativa entre espectadores fans y espectadores no fans de acuerdo con sus competencias digitales. En segundo lugar, se busca analizar el grado de correlación existente entre el nivel de competencias digitales del espectador fan y el número de series y películas del que se considera fan. En línea con el marco teórico estudiado, las competencias digitales deben estar más presentes en aquellos espectadores fans, pues se entiende, según los autores, que estos usuarios son —en potencia- más competentes digitalmente por el mero aprendizaje a través de la puesta en práctica de dichas competencias (Mihailidis, 2014; Jenkins, 2006).

\section{Metodología}

\section{Método y diseño}

La literatura de fan studies ha utilizado principalmente técnicas de estudio más cualitativas, como etnografías, focus groups o entrevistas personales. Para aproximarnos a este análisis, se ha optado por una metodología cuantitativa mediante un cuestionario enviado a un panel de consumidores de espectadores españoles. Si bien hay que tener en cuenta que, como 
indica Hills (2002, p. 66), preguntar directamente a la audiencia sobre su identidad y acciones fans no es una garantía científica de conocimiento, con el uso de esta metodología se ha pretendido aportar una nueva aproximación académica al estudio de audiencias activas. Al mismo tiempo, el trabajo pretende unificar los estudios de Mihailidis (2014) sobre competencias digitales con los fan studies y establecer un vínculo entre ambos trabajos, pues, como explica Jenkins (2006) sobre la cultura participativa: es muy importante el poder prospectivo de la alfabetización mediática para promover conductas activas. El diseño utilizado para llevar a cabo la investigación fue selectivo para el objetivo 1 y comparativo para los objetivos 2 y 3 (Ato, López y Benavente, 2013).

\section{Participantes y procedimiento}

Los datos de este estudio fueron recopilados a través de un panel de consumidores que se administró en línea en 2015. Completaron el cuestionario 1003 sujetos españoles de forma voluntaria y anónima, que cumplieron el criterio de consumir habitualmente películas y series de televisión.

El muestreo de participantes fue estratificado proporcionalmente en función del sexo y la edad (16-20 años, 20-30 años y 30-40 años) y puede considerarse que la muestra recopilada es representativa de la sociedad española, con un error asociado en torno a $3.3 \%$ y un nivel de confianza de $97 \%$.

Las características de la muestra total están disponibles en Lozano, Sánchez-Martín y Plaza (2015). Con respecto a los espectadores de la muestra que se consideran fans, estos presentan una edad media de 26.94 años $(D T=7.28)$. De estos espectadores fans, $42 \%$ tenía estudios superiores, $35 \%$ formación profesional de grado superior y $19.5 \%$ había cursado la segunda fase de la educación secundaria. Tan solo $4.5 \%$ se componía de personas sin estudios, con educación primaria o solo la primera fase de la educación secundaria. Teniendo esto en cuenta, puede afirmarse que la muestra analizada comprendía sujetos con un nivel educativo mayoritariamente superior. Respecto de la actividad profesional actual, $42.7 \%$ eran trabajadores por cuenta ajena frente a $5 \%$ por cuenta propia; $40.6 \%$ eran estudiantes de 
cualquier nivel y $11.8 \%$ eran desempleados. Por tanto, la muestra puede considerarse activa profesionalmente.

Para comprobar el segundo de los objetivos planteados, debido a la diferencia en el tamaño de la muestra de fans $(n=868) v s$. no fans $(n=135)$ en España, se seleccionó de forma aleatoria un número proporcional de espectadores (148 fans; 135 no fans). Mediante el uso del azar en la composición de los grupos de fans, se asegura estadísticamente la equivalencia entre los grupos en diversas variables.

\section{Instrumentos}

Los ítems utilizados para este trabajo forman parte de un estudio más extenso, en el que se valoraron varios constructos relacionados con la recepción de ficciones audiovisuales en el espectador español. En este caso, la información utilizada se dividió en tres partes diferenciadas. En la primera de ellas, los encuestados debían responder a preguntas de tipo sociodemográfico: edad, sexo, nivel de estudios más alto conseguido y actividad profesional actual. Otros ítems hacían referencia a si se consideraban fans de alguna película o serie de televisión, debiendo marcar sí o no en un listado proporcionado. Una última parte estaba orientada a conocer el nivel que manifestaban respecto de varios ítems relacionados con las competencias digitales. En concreto, fueron nueve ítems medidos en una escala tipo Likert de siete puntos (de totalmente en desacuerdo a totalmente de acuerdo): "Domino la búsqueda de información en internet" en ordenador portátil o de sobremesa, en móvil y en tableta (tres ítems); "Edito contenidos digitales en internet en diferentes formatos (textos, vídeos, audios e imágenes)” en ordenador portátil o de sobremesa, en móvil y en tableta (tres ítems); e "Interacciono con otros usuarios en foros, redes sociales o plataformas colaborativas (wikis, blogs) aportando información útil, editando contenidos (textos, vídeos, audios, hipervínculos e imágenes) o participando en conversaciones" en ordenador portátil o de sobremesa, en móvil y en tableta (tres ítems). Este conjunto de ítems no estaba pensado como un cuestionario para analizar el constructo de competencias digitales, sino que tenía un objetivo de naturaleza más exploratoria y basado en los diferentes paradigmas teóricos expuestos, con la intención de extraer los rasgos y las prácticas comunes de la praxis digital de los fans. 


\section{Análisis estadísticos}

Para comprobar los objetivos, en primer lugar, se analizaron las competencias digitales de los participantes de forma descriptiva mediante frecuencias y estadísticos de tendencia central (medias) y dispersión (desviaciones típicas). Posteriormente, para analizar la existencia de diferencias en el nivel de competencias digitales en función de variables como el sexo, el rango de edad, la actividad desarrollada o los estudios, se realizaron análisis no paramétricos, ya que las variables analizadas no cumplían el supuesto de normalidad; concretamente, se utilizó la prueba $\mathrm{H}$ de Kruskal-Wallis (comprueba si existen diferencias, en global, entre todos los grupos) y U de Mann-Whitney (comprueba entre qué grupo existen las diferencias detectadas previamente, si las hubiera). Para analizar la magnitud de las diferencias significativas detectadas, se utilizó el tamaño del efecto; concretamente, se calculó empleando la fórmula $\mathrm{r}=\mathrm{z} / \sqrt{ } \mathrm{n}$ en el caso de los contrastes no paramétricos (Rosenthal, 1991, citado en Field, 2009), interpretándose como tamaño de efecto pequeño si $r<0.30$, como tamaño de efecto mediano si $0.30 \leq \mathrm{r} \leq 0.50$ o como tamaño de efecto grande si $r>0.50$ (Cohen, 1992). Asimismo, se han llevado a cabo análisis de correlaciones entre las variables de tipo cuantitativo; en este caso, para comprobar el tamaño del efecto, el valor de las correlaciones obtenido se interpretó de la misma forma que anteriormente.

\section{Resultados}

\section{Objetivo 1: Descripción del espectador fan español en relación con sus competencias digitales}

De forma general, se comprueba que $85 \%$ de los participantes está muy de acuerdo con que domina la búsqueda de información en internet a través de ordenador portátil o de sobremesa, $70.5 \%$ a través de móvil y $56 \%$ a través de tableta. El 34 \% está muy de acuerdo con que edita contenidos digitales en internet en diferentes formatos (textos, vídeos, audios e imágenes) a través de ordenador portátil o de sobremesa, $15 \%$ a través de móvil y $9.5 \%$ a través de tableta. El $22 \%$ está muy de acuerdo con que interacciona con otros usuarios en foros, redes sociales o plataformas colaborati- 
vas (wikis, blogs) aportando información útil, editando contenidos (textos, vídeos, audios, hipervínculos e imágenes) o participando en conversaciones a través de ordenador portátil o de sobremesa, $21 \%$ a través de móvil y $10 \%$ a través de tableta.

A continuación, en la tabla 1, se aportan las medias y desviaciones típicas de los diferentes ítems de competencias digitales. Como puede observarse, la práctica general que dominan los espectadores fans es la búsqueda de información, independiente de su soporte (ordenador, móvil y tabletas). Tras esto, la edición de contenidos en ordenador y la interacción en ordenador y móviles y, por último, la edición o producción de contenidos en móviles y tabletas.

\section{Tabla 1. El nivel medio en competencias digitales}

\begin{tabular}{|c|c|c|c|c|c|c|c|c|c|}
\hline \multirow{2}{*}{} & \multicolumn{3}{|c|}{ Ordenador } & \multicolumn{3}{c|}{ Móvil } & \multicolumn{3}{c|}{ Tableta } \\
\cline { 2 - 12 } & Búsqueda & Edición & Interacción & Búsqueda & Edición & Interacción & Búsqueda & Edición & Interacción \\
\hline M & 6.39 & 3.74 & 3.31 & 5.93 & 2.79 & 3.14 & 5.04 & 2.21 & 2.28 \\
\hline DT & 1.17 & 2.46 & 2.23 & 1.53 & 2.07 & 2.20 & 2.24 & 1.87 & 1.89 \\
\hline
\end{tabular}

Fuente: Elaboración propia.

Respecto de las diferencias en el nivel de competencias digitales en función del sexo, se comprobó la existencia de diferencias significativas entre hombres y mujeres en el nivel de competencia para interaccionar con otros usuarios en foros, redes sociales o plataformas colaborativas a través de ordenador $(z=-3.18, p=0.001, r=-0.11)$. Los hombres manifestaron mayores puntuaciones que las mujeres, si bien es cierto que, a nivel medio, tampoco fueron muy diferentes (mujer: $\mathrm{M}=3.06$, DT $=2.20$; hombre: $\mathrm{M}=3.55, \mathrm{DT}=2.24)$.

En cuanto a las diferencias en el nivel de competencias digitales en función del rango de edad, se encontró que existían diferencias estadísticamente significativas en todas las variables, excepto en las tres que hacen referencia al uso de tableta. Concretamente, como se muestra en la tabla 2 , en el dominio en la búsqueda de información en internet a través de ordenador las diferencias están entre las edades 16-20 y 31-40 y entre 21-30 
y 31-40. En la edición de contenidos digitales en internet a través de ordenador, las diferencias están entre las edades $16-20$ y 31-40 y entre 21-30 y 31-40, y mediante móvil las diferencias están entre las edades $16-20$ y 31 40. En la interacción con otros usuarios a través de ordenador, las diferencias están entre las edades $16-20$ y 31-40, y a través de móvil las diferencias están entre las edades 16-20 y 31-40.

\section{Tabla 2. Diferencias en competencias digitales en función del rango de edad}

\begin{tabular}{|c|c|c|c|c|c|c|c|c|}
\hline \multirow{2}{*}{ Ítems } & \multicolumn{2}{|c|}{ H de Kruskal-Wallis } & \multicolumn{3}{|c|}{ Grupos } & \multicolumn{3}{|c|}{ U de Mann-Withney } \\
\hline & $X^{2}(2)$ & $\mathrm{p}$ & Edad & M & DT & $\mathbf{z}$ & $\mathrm{p}$ & $\mathbf{r}$ \\
\hline \multirow{4}{*}{$\begin{array}{c}\text { Búsqueda de información } \\
\text { en internet a través de } \\
\text { ordenador }\end{array}$} & \multirow{4}{*}{15.56} & \multirow{4}{*}{0.000} & $16-20$ & 6.44 & 1.19 & \multirow{2}{*}{-3.21} & \multirow{2}{*}{0.001} & \multirow{2}{*}{-0.13} \\
\hline & & & $31-40$ & 6.21 & 1.25 & & & \\
\hline & & & $21-30$ & 6.51 & 1.04 & \multirow{2}{*}{-3.51} & \multirow{2}{*}{0.000} & \multirow{2}{*}{-0.15} \\
\hline & & & $31-40$ & 6.21 & 1.25 & & & \\
\hline \multirow{4}{*}{$\begin{array}{l}\text { Edición de contenidos } \\
\text { digitales en internet a } \\
\text { través de ordenador }\end{array}$} & \multirow{4}{*}{23.96} & \multirow{4}{*}{0.000} & $16-20$ & 4.09 & 2.41 & \multirow{2}{*}{-4.66} & \multirow{2}{*}{0.000} & \multirow{2}{*}{-0.19} \\
\hline & & & $31-40$ & 3.15 & 2.40 & & & \\
\hline & & & $21-30$ & 3.94 & 2.47 & \multirow{2}{*}{-3.78} & \multirow{2}{*}{0.000} & \multirow{2}{*}{-0.16} \\
\hline & & & $31-40$ & 3.15 & 2.40 & & & \\
\hline \multirow{2}{*}{$\begin{array}{l}\text { Edición de contenidos } \\
\text { digitales en internet a } \\
\text { través de móvil }\end{array}$} & \multirow{2}{*}{22.58} & \multirow{2}{*}{0.000} & $16-20$ & 3.21 & 2.12 & \multirow{2}{*}{-4.62} & \multirow{2}{*}{0.000} & \multirow{2}{*}{-0.19} \\
\hline & & & $31-40$ & 2.45 & 2.00 & & & \\
\hline \multirow{2}{*}{$\begin{array}{l}\text { Interacción con otros } \\
\text { usuarios a través de } \\
\text { ordenador }\end{array}$} & \multirow{2}{*}{12.20} & \multirow{2}{*}{0.002} & $16-20$ & 3.60 & 2.25 & \multirow{2}{*}{-3.47} & \multirow{2}{*}{0.001} & \multirow{2}{*}{-0.14} \\
\hline & & & $31-40$ & 2.99 & 2.19 & & & \\
\hline $\begin{array}{l}\text { Interacción con otros } \\
\text { usuarios a través de móvil }\end{array}$ & 6.75 & 0.034 & $16-20$ & 3.36 & 2.20 & -2.52 & 0.012 & -0.10 \\
\hline
\end{tabular}

Fuente: Elaboración propia.

Haciendo alusión a la actividad que desarrollan los participantes, y como se aprecia en la tabla 3, se encuentran diferencias en el nivel de competencias digitales en el dominio en la búsqueda de información en internet a través de ordenador, las cuales están entre los estudiantes y los trabajadores por cuenta ajena y entre estudiantes y desempleados. También se encuentran diferencias significativas en la edición de contenidos a través de ordenador, las cuales están entre los estudiantes y los trabajadores por cuenta ajena y entre estudiantes y desempleados. 


\section{Tabla 3. Diferencias en el nivel de competencias digitales en función de la actividad}

\begin{tabular}{|c|c|c|c|c|c|c|c|c|}
\hline \multirow[t]{2}{*}{ Ítems } & \multicolumn{2}{|c|}{ H de Kruskal-Wallis } & \multicolumn{3}{|c|}{ Grupos } & \multicolumn{3}{|c|}{ U de Mann-Withney } \\
\hline & $X^{2}(3)$ & $\mathrm{p}$ & Actividad & M & DT & $\mathrm{z}$ & $\mathrm{p}$ & $\mathbf{r}$ \\
\hline \multirow{4}{*}{$\begin{array}{l}\text { Dominio en la búsqueda } \\
\text { de información en internet } \\
\text { a través de ordenador }\end{array}$} & \multirow{4}{*}{11.92} & \multirow{4}{*}{0.008} & Estudiantes & 6.52 & 1.10 & -3.30 & 0.001 & -0.12 \\
\hline & & & TCA & 6.32 & 1.17 & & & \\
\hline & & & Estudiantes & 6.52 & 1.10 & -2.07 & 0.038 & -0.10 \\
\hline & & & Desempleados & 6.35 & 1.01 & & & \\
\hline \multirow{4}{*}{$\begin{array}{l}\text { Edición de contenidos a } \\
\text { través de ordenador }\end{array}$} & \multirow{4}{*}{10.35} & \multirow{4}{*}{0.016} & Estudiantes & 4.05 & 2.42 & -2.45 & 0.014 & -0.09 \\
\hline & & & TCA & 3.60 & 2.51 & & & \\
\hline & & & Estudiantes & 4.05 & 2.42 & -2.72 & 0.006 & -0.13 \\
\hline & & & Desempleados & 3.28 & 2.43 & & & \\
\hline
\end{tabular}

TCA: trabajo por cuenta ajena.

Fuente: Elaboración propia.

Atendiendo al nivel de estudios (educación secundaria, formación profesional/grado superior/bachillerato, educación superior), no se han detectado diferencias significativas en ninguna de las variables relacionadas con las competencias digitales en función del nivel de estudios. Concretamente, los resultados obtenidos se muestran en la tabla 4 .

\section{Tabla 4. Diferencias en competencias digitales en función del nivel de estudios}

\begin{tabular}{|c|c|c|}
\hline \multirow{2}{*}{ Ítems } & \multicolumn{2}{|c|}{ H de Kruskal-Wallis } \\
\cline { 2 - 3 } & $\boldsymbol{X}^{2}(\mathbf{2})$ & $\mathrm{p}$ \\
\hline Búsqueda a través de ordenador & 0.90 & 0.638 \\
\hline Edición a través de ordenador & 0.11 & 0.947 \\
\hline Interacción a través de ordenador & 0.40 & 0.946 \\
\hline Búsqueda a través de móvil & 0.11 & 0.232 \\
\hline Edición a través de móvil & 2.92 & 0.531 \\
\hline Interacción a través de móvil & 1.27 & 0.496 \\
\hline Búsqueda a través de tableta & 1.40 & 0.643 \\
\hline Edición a través de tableta & 0.88 & 0.851 \\
\hline Interacción a través de tableta & 0.32 & \\
\hline
\end{tabular}

Fuente: Elaboración propia. 


\section{Objetivo 2: Comparativa de fans y no fans respecto del nivel de competencias digitales}

Teniendo en cuenta la muestra total de 1003 espectadores españoles, 86.5\% (868 sujetos) se considera fan de alguna película o serie de televisión frente a $13.5 \%$ (135 sujetos) que declara no ser fan de ninguna. Centrándonos en los datos, una vez realizamos la selección aleatoria sobre los fans y trabajando, por tanto, con un número similar de fans y no fans, podemos afirmar, como se muestra en la tabla 5, que existen diferencias estadísticamente significativas respecto de la búsqueda de información a través de ordenador y a través de móvil, así como de la interacción en línea a través de ordenador y a través de móvil.

Tabla 5. Comparativa de fans y no fans respecto del nivel de competencias digitales

\begin{tabular}{|c|c|c|c|c|c|c|}
\hline \multirow{2}{*}{ Ítems } & \multicolumn{3}{|c|}{ Grupos } & \multicolumn{3}{|c|}{ U de Mann-Withney } \\
\hline & fans/no fans & M & DT & $\mathrm{z}$ & $\mathrm{p}$ & $\mathbf{r}$ \\
\hline \multirow{2}{*}{ Búsqueda de información a través de ordenador } & Fans & 6.32 & 1.28 & \multirow{2}{*}{-3.12} & \multirow{2}{*}{0.002} & \multirow{2}{*}{-0.19} \\
\hline & No fans & 5.88 & 1.53 & & & \\
\hline \multirow{2}{*}{ Búsqueda de información a través de móvil } & Fans & 5.90 & 1.62 & \multirow{2}{*}{-3.22} & \multirow{2}{*}{0.001} & \multirow{2}{*}{-0.19} \\
\hline & No fans & 5.30 & 1.81 & & & \\
\hline \multirow{2}{*}{ Interacción en línea a través de ordenador } & Fans & 3.28 & 2.23 & \multirow{2}{*}{-2.50} & \multirow{2}{*}{0.013} & \multirow{2}{*}{-0.15} \\
\hline & No fans & 2.64 & 2.04 & & & \\
\hline \multirow{2}{*}{ Interacción en línea a través de móvil } & Fans & 2.99 & 2.13 & \multirow{2}{*}{-2.45} & \multirow{2}{*}{0.014} & \multirow{2}{*}{-0.15} \\
\hline & No fans & 2.41 & 1.91 & & & \\
\hline
\end{tabular}

Fuente: Elaboración propia.

\section{Objetivo 3: Correlación entre el nivel de competencias digitales y el número de series y películas del que se consideran fans}

Utilizando la correlación de Pearson, se encontraron relaciones significativas y positivas entre el número de series y películas del que se consideran fans y las variables relacionadas con las competencias digitales a través de los tres dispositivos, a excepción de la búsqueda de información. Concretamente, con la edición $(r=0.14, p=0.000)$ y la interacción en línea $(r=0.19$, $p=0.000)$ mediante ordenador, con la edición $(r=0.11, p=0.002)$ y la 
interacción en línea $(r=0.17, p=0.000)$ mediante móvil, así como con la edición $(r=0.11, p=0.002)$ y la interacción en línea $(r=0.13, p=0.000)$ mediante tableta. Estos resultados indican que, a mayor número de series de las que se consideran fans, mayor es el nivel de competencias digitales (a excepción de la búsqueda de información), si bien es cierto que el tamaño del efecto fue pequeño en todos los casos $(r<0.30)$.

\section{Discusión y conclusiones}

En primer lugar, de acuerdo con los resultados obtenidos y respecto de la radiografía de competencias digitales del espectador fan, de las tres competencias analizadas en cada uno de los tres dispositivos tecnológicos, la práctica general que dominan los espectadores fans es la búsqueda de información. Además, como se destacó, la edición o producción de contenidos en móviles y tabletas parece ser la competencia menos autopercibida por los fans.

Si tenemos en cuenta las competencias digitales de estos espectadores, se observa una brecha digital generacional entre los espectadores más jóvenes, que se consideran más competentes en la búsqueda de información en ordenador y en la edición de contenidos y la interacción con otros usuarios en internet mediante ordenador y móvil. Igualmente, y atendiendo a la actividad, los estudiantes son los que dominan mejor la búsqueda de información y la edición de contenidos en internet a través de ordenador. Estos datos están en la línea de los ofrecidos por el INE (2016) y un reciente trabajo sobre la brecha digital entre adultos y adolescentes en España (Garrido-Lora, Busquet y Munté-Ramos, 2016). Este último trabajo coincide con los datos aquí obtenidos subrayando que existen efectivamente diferencias importantes en el uso y dominio de las tecnologías de la información y de la comunicación, haciendo los jóvenes un uso más intenso de ellas que los adultos.

En segundo lugar, los resultados demuestran que el espectador fan tiene mayores competencias digitales que el espectador no fan. Estas diferencias significativas en la comparativa se reflejan en las competencias de búsqueda de información e interacción, tanto en ordenadores como en dispositivos móviles, dejando de lado la tableta. De acuerdo con los resulta- 
dos, el uso de la tableta y la competencia digital de edición de contenidos parecen no ser competencias que definan y diferencien a los espectadores fans, que manifiestan los mismos niveles de desarrollo en estas competencias que los no fans. Estos resultados extraídos en España coinciden con los estudios señalados más arriba (Bourdaa y Lozano Delmar, 2016; Cascajosa, 2016; Jenkins, 2014; Mittell, 2013; Zubernis y Larsen, 2012). Es decir, los fans se diferenciarían de los no fans en aquellas competencias digitales más vinculadas a la búsqueda de información y la socialización e interacción, en mayor medida que aquellas relacionadas con la producción o edición de contenido. Teniendo esto en cuenta, los fans se ubicarían, dentro del continuum de Abercrombie y Longhurst (1998), entre los fans y los enthusiasts.

Dicho en otras palabras, podría decirse que la mayoría de los espectadores que se consideran fans justifican esta identidad con prácticas vinculadas al viewing y el acting, antes que al producing o la generación de contenidos (Bourdaa y Lozano, 2016, p. 12). Es decir, la inmersión en los productos ficcionales, los modos de consumo colectivo y conectado a través de la televisión social, las experiencias compartidas y los contenidos "propagables" parecen efectivamente tener más peso en la definición de las identidades de los fans. Así pues, estas competencias digitales contribuirían también a tres de las praxis digitales comentadas por Bennett (2014): 1) comunicación entre comunidades, 2) conocimiento compartido y 3) empoderamiento ciudadano, quedando la creatividad mediante la edición de contenido (artworks, fanfics, performing songs, etc.) en un segundo lugar.

Por otra parte, los bajos resultados obtenidos en las competencias digitales de uso de tabletas pueden explicarse si se tiene en cuenta la penetración de las tabletas en la población española. Según datos de la AIMC (2016), 59.1\% de los usuarios accede a internet a través de tabletas frente a $93.9 \%$ que lo hace a través del teléfono móvil. Según la Sociedad de la Información en España 2015 (Fundación Telefónica, 2016, p. 67), "el ordenador domina el uso profesional y el ocio, el smartphone la comunicación, la tablet complementa a estos dos dispositivos". Otra posible razón tiene que ver con el distinto uso que se realiza de estos dispositivos, ya que el teléfono móvil podría ser usado de una manera más activa (elaboración y modi- 
ficación de contenidos) frente a un uso más pasivo de la tableta (consumo de contenidos).

En tercer lugar, y en respuesta a otro de los objetivos de la investigación, el nivel de competencias digitales en los ítems de interacción y edición de contenidos en los espectadores fans es directamente proporcional al número de productos de los que el espectador se considera fan. Es importante destacar que la competencia de la búsqueda de información no mejora, ya que se encontraba en niveles muy altos de partida tanto en espectadores fans como no fans. Además, de acuerdo con los datos obtenidos, no existen diferencias significativas en cuanto al nivel de competencias digitales de los fans según su nivel de estudios. Por tanto, podría afirmarse que los fans entrenan y mejoran sus competencias digitales poniendo en práctica sus actividades fans. Estos resultados son similares a los obtenidos en otro estudio que demostraba que ser fan también contribuía al entrenamiento y mejora de las fortalezas y virtudes asociadas a la felicidad eudaimónica (Lozano, Sánchez-Martín y Muñiz, 2016).

Teniendo en cuenta estos resultados, y como conclusión, en consonancia con aquello que afirman Bennet (2014), Mihailidis (2014) y Jenkins (2006), la alfabetización mediática, es decir, el entrenamiento de las competencias digitales, hace que estas mejoren. Así pues, un sujeto será más hábil en sus contribuciones en línea en función de la praxis, esto es, del ejercicio y la puesta en práctica de la actividad digital. Por otro lado, el resultado de la praxis tendrá como consecuencia, atendiendo igualmente al razonamiento de los expertos, un efecto de empoderamiento de las audiencias activas por su capacidad para crear contenidos dentro del ecosistema mediático. Por lo que podemos afirmar que, además, el espectador fan es más poderoso que el espectador no fan.

Por último, esta investigación nos ofrece una oportunidad para continuar explorando sobre los fans y su impacto en cuanto es un público formado y empoderado que contribuye con su actividad a la construcción de mensajes. Pues, si como se ha demostrado, los fans dominan las competencias digitales de búsqueda de información e interacción mejor que 
los espectadores no fans, estas prácticas pueden ser aprovechadas respecto del compromiso, la mejora, la ampliación o el enriquecimiento de productos y así responder a la cultura de la colaboración de la que forman parte.

\section{Referencias}

Abercrombie, N. y Longhurst, B. J. (1998). Audiences: A sociological theory of performance and imagination. Londres: Sage.

Aced, C. (2010). Redes sociales en una semana. Barcelona: Gestión 2000.

Aguaded, J. I. y Urbano-Cayuela, R. (2014). Nuevo modelo de enseñanza europeo a través del prosumidor infantil televisivo. Comunicación $y$ Hombre, 10, 131-142.

AIMC (Asociación para la Investigación de Medios de Comunicación) (2016). $18^{\circ}$ Navegantes en la Red. Recuperado de http://www. aimc.es/-Infografia-18 \%C2 \%AA-Encuesta-AIMC-a-html

Ato, M., López, J. J. y Benavente, A. (2013). A classification system for research designs in psychology. Anales de Psicología, 29(3), 1038-1059.

Bennett, L. (2014). Tracing textual poachers: Reflections on the development of fan studies and digital fandom. The Journal of Fandom Studies, 2(1), 5-20. http://doi.org/10.1386/jfs.2.1.5_1

Bergsma, L., Considine, D., Culver, S. H., Hobbs, R., Jensen, A., Rogow, F., Rosen, E., Scheibe, C., Sellers-Clark, S. y Thoman, E. (2007). Core principles of media literacy education. Denver, CO: Alliance for a Media Literate America.

Bourdaa, M. y Lozano Delmar, J. (2016). Contemporary participative TV audiences: Identity, authorship and advertising practices between fandom. Participations. Journal of Audience and Reception Studies, 13(2), 2-13. 
Cascajosa Virino, C. (2016). La cultura de las series. Barcelona: Laertes.

Cobo Romaní, C. y Pardo Kuklinski, H. (2007). Planeta Web 2.0. Inteligencia colectiva o medios fast food. Barcelona: Grup de Recerca d'Interaccions Digital.

Cohen, J. (1992). A power primer. Psychological Bulletin, 112(1), 155-159. http://dx.doi.org/10.1037/0033-2909.112.1.155

Dans, E. (2010). Todo va a cambiar. Barcelona: Deusto.

Dhar, V. y Chang, E. A. (2009). Does chatter matter? The impact of usergenerated content on music sales. Journal of Interactive Marketing, 23(4), 300-307.

Ferrés i Prats, J., Aguaded-Gómez, I. y García-Matilla, A. (2012). La competencia mediática de la ciudadanía española: dificultades y retos. Icono14, 10(3), 23-42. doi: 10.7195/ri14.v10i3.201

Ferrés i Prats J. y Masanet, M. J. (2015). A vueltas con la educación mediática. En J. Ferrés i Prats y J. M. Masanets (eds.), La educación mediática en la universidad española (pp. 13-29). Barcelona: Gedisa.

Field, A. (2009). Discovering statistic using SPSS (And sex, and drugs and rock ' $n$ ' roll). Londres: Sage.

Fiske, J. (1992). The cultural economy of fandom. En L. A. Lewis (ed.), The adoring audience: Fan culture and popular media (pp. 30-49). Londres: Routledge.

Frau-Meigs, D. (2014). Derechos humanos y educación mediática en la era ciberista. UNED. Recuperado de https://goo.gl/NhYwnp

Fundación Telefónica (2016). La Sociedad de la Información en España 2015. Recuperado de https://goo.gl/hNL96g 
Garrido-Lora, M., Busquet Duran, J. y Munté-Ramos, R. A. (2016). Estudio sobre el uso de las TIC y la brecha digital entre adultos y adolescentes en España. Anàlisi. Quaderns de Comunicació I Cultura, 54, 44-57.

Grandío, M. (2016). Adictos a las series: 50 años de lecciones de los fanes. Barcelona: UOC.

Gray, J., Harrington, C. L. y Sandvoss, C. (2007). Fandom: Identities and communities in a mediated world. Nueva York: New York University Press.

Guerrero, M. (2015). Producción y lectura de fan fiction en la comunidad online de la serie Fringe: transmedialidad, competencia y alfabetización mediática. Palabra Clave, 18(3), 722-745. doi: 10.5294/ pacla.2015.18.3.5

Hays, S., Page, S. J. y Buhalis, D. (2013). Social media as a destination marketing tool: Its use by national tourism organizations. Current Issues in Tourism, 16(3), 211-239.

Hellekson, K. y Busse, K. (2014). The fanfiction studies reader. Iowa: University of Iowa Press.

Hills, M. (2002). Fan cultures. Londres: Routledge.

Hills, M. (2013). Fiske's “textual productivity” and digital fandom: Web 2.0 democratization versus fan distinction? Participations: Journal of Audience y Receptions Studies, 10(1), 130-153. Recuperado de https://goo.gl/5jeSsA

INE (Instituto Nacional de Estadística) (2016). Encuesta sobre equipamiento y uso de tecnología de la información y la comunicación en los hogares. Recuperado de https://goo.gl/g9d2cb 
Islas, O. y Arribas Urrutia, A. (2010). Comprender las redes sociales como ambientes mediáticos. En A. Piscitelli, I. Adaime e I. Binder (eds.), El proyecto Facebook y la posuniversidad (pp. 147-161). Madrid: Ariel.

Jenkins, H. (1992). Textual poachers: Television fans and participatory culture. Nueva York, NY: Routledge.

Jenkins, H. (2006). Convergence culture: Where new and old media collide. Nueva York, NY: New York University Press.

Jenkins, H. (2014). Where fandom studies came from: An interview with Kristina Busse and Karen Hellekson (Part Two) [Weblogpost]. Recuperado de https://goo.gl/UvtZoA

Jenkins, H., Ford, S. y Green, J. (2015). Cultura transmedia: la creación de contenido y valor en una cultura en red. Barcelona: Gedisa.

Kaplan, A. M. y Haenlein, M. (2010). Users of the world, unite! The challenges and opportunities of Social Media. Business Horizons, 53(1), 59-68.

Kietzmann, J. H., Hermkens, K., McCarthy, I. P. y Silvestre, B. S. (2011). Social media? Get serious! Understanding the functional building blocks of social media. Business Horizons, 54(3), 241-251.

Lewis, L. A. (1992). The adoring audience: Fan culture and popular media. Nueva York: Routledge.

Lozano Delmar, J., Sánchez-Martín, M. y Muñiz Velázquez, J. A. (2016). To be a fan is to be happier: Using the eudaimonic spectator questionnaire to measure eudaimonic motivations in spanish fans. Journal of Happiness Studies. doi:10.1007/s10902-016-9819-9

Lozano Delmar, J., Sánchez-Martín, M. y Plaza, J. F. (2015). Portrait Robot d'un fan Espagnol: Analyse sociodémographique et habitudes 
de consommation chez le fan de films et de séries télé en Espagne. Revue française des sciences de l'information et de la communication, 7. doi: 10.4000/rfsic. 1692

Mihailidis, P. (2014). Media literacy and the emerging citizen: Youth, engagement and participation in digital culture. Nueva York: Peter Lang.

Mittell, J. (2013). Forensic fandom and the drillable text. Recuperado de https://goo.gl/w1iBDY

O'Reilly, T. (2005). What is web 2.0? Design patterns and business models for the next generation of software. Recuperado de https:// goo.gl/2XyABK

Pearson, R. (2010). Fandom in the digital era. Popular Communication, 8(1), 84-95. http://doi.org/10.1080/15405700903502346

Quintas Froufe, N. y González Neira, A. (coord.) (2015). La participación de la audiencia en la televisión: de la audiencia activa a la social. Madrid: AIMC. Recuperado de https://goo.gl/DBsevR

Ransbotham, S., Kane, G. C. y Lurie, N. H. (2012). Network characteristics and the value of collaborative user-generated content. Marketing Science, 31(3), 387-405.

Ritzer, G. y Jurgenson, N. (2010). Production, consumption, prosumption: The nature of capitalism in the age of the digital "prosumer". Journal of Consumer Culture, 10(1), 13-36.

Rosenthal, R. (1991). Meta-analytic procedures for social research. Newbury Park, CA: Sage.

Sandvoss, C. (2005). Fans: The mirror of consumption. Cambridge: Polity Press. 
Shirky, C. (2010). Cognitive surplus: How technology makes consumers into collaborators. Nueva York: Penguin.

Toffler, A. y Toffler, H. (1980). The third wave. Nueva York: William Marrow.

Van Dijck, J. (2009). Users like you? Theorizing agency in user- generated content. Media, Culture y Society, 31(1), 41-58.

Zubernis, L. y Larsen, K. (2012). Fandom at the crossroads: Celebration, shame and fan/producer relationships. Newcastle upon Tyne, Reino Unido: Cambridge Scholars. 